\title{
A METHOD OF MEASURING THE SUSCEPTIBILIY OF WEAKLY MAGNETIC SUBSTANCES AND A STUDY OF THE SUSCEPTIBILITY OF ALLOYS OF BISMUTH WITH TELLURIUM AND THALLIUM.
}

By C. E. Mendenhall and W. F. Lent.

IN the Physical Review, Volume XXVI., Number 6, Clifford describes a method for measuring susceptibility which gave excellent results but which involved the rather tedious determina-

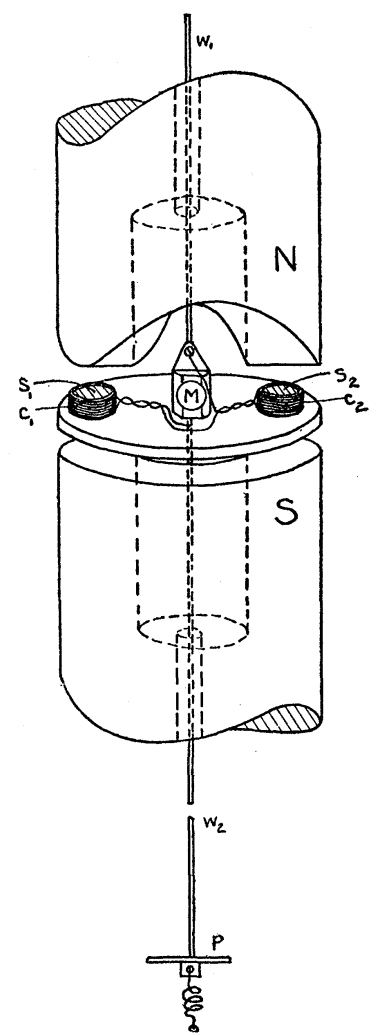

Fig. 1. tion of the magnetic field-intensity throughout a region in which the specimen under test was to beused, in order that the equation

$$
f_{x}=\frac{d H}{d x} H k V
$$

might be used. In this equation, $H$ is the magnetic field-intensity, $d H / d x$, its rate of change along $x ; k$, the susceptibility and $\bar{V}$ the volume of the specimen, while $f_{x}$ is the resulting ponderamotive force, along $x$, on the specimen. It occurred to one of us that considerable simplification, and possibly some increase in accuracy, would result if the arrangements were changed so as to give virtually a "zero" method. The present paper gives an account of the new method, together with the results of a study of the series of bismuth-tellurium and bismuth-thallium alloys, and some preliminary observations on the effects of heat treatment on the susceptibility of bismuth.

The principle of this method, which is applicable equally well to measurement of 
para- and dia-magnetic bodies, is, briefly, to balance the torque produced by the action of a non-uniform magnetic field upon two symmetrically-placed specimens of the substance under test, by the torque due to the action of the same field upon coils immediately surrounding the specimens, the current in which can be measured. The general arrangement of the apparatus will be clear from Fig. I, where $N, S$ are the poles of the magnet producing the testing field; $S_{1} S_{2}$, the small cylinders of the metal under test; $c_{1} c_{2}$, the cylindrical coils accurately surrounding the test specimens, through which the balancing current is passed and $w_{1} w_{3}$ the suspending wires which also serve to carry the current to and from the coils $c$.

In order to bring out the quantitative conditions on which the method rests, consider a section through the (circular) specimens by a plane perpendicular to the axis of suspension, Fig. 2, where $r$

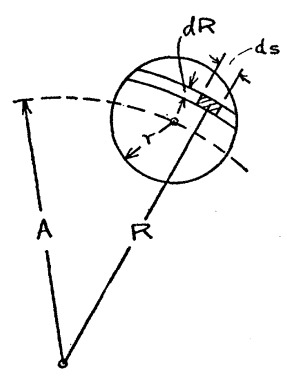

Fig. 2.

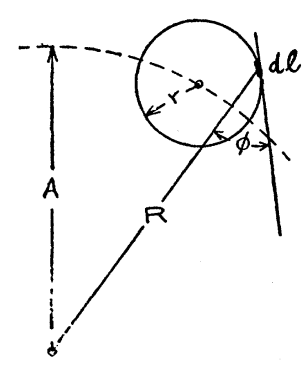

Fig. 3.

is the radius of the specimen, and also of the single layer coil surrounding it; $R$, the distance from the axis of suspension to an element of the circumference. The expression for the moment of the forces acting upon a thin section of the specimen, cut parallel to the plane of the paper, around an axis through $O$ perpendicular to the plane of the paper is:

$$
\begin{aligned}
M_{S} & =d t \int_{A-r}^{A+r} R \int_{H_{1}}^{H_{2}} k H d R \frac{d H}{d S} d S \\
& =k d t \int_{A-r}^{A+r} R d R \frac{\left(H_{2}+H_{1}\right)}{2}\left(H_{2}-H_{1}\right),
\end{aligned}
$$

where $d t$ is the thickness of the section and $H_{2}, H_{1}$ are the com- 
ponents of the magnetic field perpendicular to the section, at the ends of the elementary strip of width, $d R$.

Similarly (Fig. 3 ) for the coil of radius $r, n$ turns per centimeter of thickness $(d t)$ and carrying a current $i$ the moment around $o$ is:

(2) $M_{c}=i n d t \int_{\Delta-r}^{A+r} R d l \cos \phi\left(H_{2}-H_{1}\right)=i n d t \int_{A-r}^{A+r} R d R\left(H_{2}-H_{1}\right)$.

If, now, $\left(H_{2}+H_{1}\right)$ is independent of $R$ we have for the balanced case when $M_{s}=M_{c}$,

$$
k \frac{H_{2}+H_{1}}{2}=i n
$$

It is evidently necessary, then, that the specimens should as nearly as possible fill the coils and that the mean field $\frac{H_{2}+H_{1}}{2}$ should not vary with $R$. Furthermore, if $\frac{H_{2}+H_{1}}{2}$ is to be determined, as is most convenient, by using the suspended coils as test coils in connection with a standardized fluxmeter, it is necessary that the lateral variations of $H$ should be practically linear, in order that the mean value of $H$ over the area of the specimen, as measured by the fluxmeter, should equal the mean value around the boundary of the specimen. Our specimens were turned to fit the coils to about $0.02 \mathrm{~mm}$., so that any error introduced hereby is negligible. Since two symmetrical coils are used it is to be observed that the $H$ and $n$ occurring in (3) are the means for the two coils.

The mean value of $H$ was determined by connecting the two coils in series with a Grassot fluxmeter and determining the deflection produced by reversing the magnetic field $;^{1}$ the fluxmeter having been calibrated in the usual way with a standard solenoid.

The expression for $H$ is then:

$$
H=\frac{\mathrm{I}}{2} \frac{d}{A N a},
$$

where: $d$ = deflection observed on reversing $H$ through coils;

${ }^{1}$ This reversal method gives correct results in the present case because of the narrow hysteresis loop due to the large air-gap. 


$$
\begin{aligned}
& A=\text { constant of fluxmeter; } \\
& N=2 n h=\text { total number of turns on coils I and } 2(h= \\
& \quad \text { height of coils); } \\
& a=\text { area of one coil. }
\end{aligned}
$$

So that

$$
\begin{aligned}
k & =\frac{A}{d} N a \cdot \frac{N}{2 h} \cdot i, \\
& =\frac{A N^{2} a^{2} i}{d V_{c}} . \\
\left(V_{c}\right. & =\text { volume of both coils. } \\
& =2 h a .)
\end{aligned}
$$

The actual test pieces used were not simple cylinders as above assumed, but had small projections on top for convenience in handling; the magnetic torque produced by them would therefore be $V_{s} / V_{c}$ times that produced by specimens just filling the coils, where $V_{s}=$ actual volume of specimens, and $V_{c}=$ volume of coils. The expression for $k$ should therefore be multiplied by $V_{c} / V_{s}$ giving, finally, $k=A N^{2} a^{2} i / d V_{s}$. $V_{s}$ was determined from the loss of weight in water.

In working this out practically the general procedure was first to adjust the torsion head, with no field on, and no specimen in place, but with equal counter-weights in the pan, $P$, below, until the coils were in the proper part of the field, this zero position being located by a scale reading reflected from the mirror $M$ of the suspended system. The counter-weights which were removed when the specimens were put in place were to avoid any error due to twisting of the suspension caused by the change in tension which would otherwise occur. The specimens were then put in place and the field put on, and the current determined which was necessary to bring the suspended system back to the zero position. ${ }^{1}$ The suspended system was then clamped in the zero position, the coils (through the suspension) disconnected from the current circuit and connected to the fluxmeter and the field $H$ determined by reversals. In determining $H$ it was found necessary to make two observations-one with the

${ }^{1}$ To eliminate any slight asymmetry of specimens and coils readings were also taken with specimens interchanged. 
coils in series with the fluxmeter, as described, and the other with only the upper and lower suspension in series with each other and the fluxmeter-i.e., the coils themselves cut out of circuit by a very small switch on the suspended system. By this means allowance could be made for the change in flux (on reversal of $H$ ) in all parts of the circuit other than the coils themselves. It was also necessary to make "blank" tests of the magnetic deflection obtained with no specimens in position - in order to correct for the magnetic properties of the suspended system. Experience emphasized the necessity for placing the counter-weights some distance below the magnet in order to avoid the torque due to stray fields.

A tantalum ribbon served excellently as an upper suspension, with a coiled phosphor-bronze ribbon for the lower one. The coils $c_{1}$ and $c_{2}$ were made by winding no. 36 enamel insulated copper wire on a carefully turned brass rod, coating with plaster of Paris and removing. The difference between the mean radius of the coil and the outside radius of the specimen was only the radius of the insulated wire, $0.07 \mathrm{~mm}$., plus $0.01 \mathrm{~mm}$. to permit the easy removal of the specimens. The diameter of the coils was measured with a micrometer microscope. The dimensions of the moving system were:

Diameter of coils $=0.8 \mathrm{I} 3 \mathrm{I} \mathrm{cm}$.,

Height of coils $=3.5 \mathrm{~mm}$. (approximately),

Distance from axis of suspension to center of coils $=2 \mathrm{~cm}$.

Total number of turns per coil $=25$.

In order to minimize the radial variation of $H$, and to produce an approximately linear transverse variation, the end of the upper hollow cylindrical pole-piece was given an approximately helicoidal shape, the air-gap varying with $\theta$ but not with $R$. The width of the annular face along $R$ was about twice the diameter of the specimens. Errors in the results due to variations in $H$ different from those assumed would in all probability be different for saturated and unsaturated pole-pieces; hence determinations of the susceptibility of bismuth were made with different magnetizing currents (saturated and unsaturated pole-pieces). As may be seen from the observations on a single specimen of bismuth grouped in 
Table I., the relations between $k$ and the exciting current $(H)$ is:

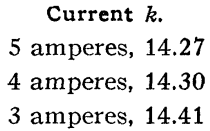

There is apparently a tendency to get larger values of $k$ with smaller field strengths-the maximum variation of these means being I per cent. It is doubtful whether this variation is really due to a departure of the field from the linear variation assumed, for it will be noticed that the accidental variations are fully as large as this. All readings except those here given were made with four amperes field current; and it would seem a reasonable conclusion from these figures of Table I. that errors of somewhat less than I per cent. are as great as need be expected in the means of a number of observations.

TABLE I.

Susceptibility of Bismuth.

Volume, 0.377 c.c. $A=2.965 \times 19^{-5}$.

\begin{tabular}{|c|c|c|c|c|c|c|c|}
\hline$I$ & $\begin{array}{r}-i c_{1} \\
\times 10^{2}\end{array}$ & $\begin{array}{r}-i_{s_{1}} \\
\times 10^{2}\end{array}$ & $\begin{array}{l}-i_{s_{2}} \\
\times \mathrm{IO}^{2}\end{array}$ & $\begin{array}{l}-i c_{2} \\
\times \mathbf{r o}^{2}\end{array}$ & $-i \times 10^{2}$ & $d$ & $-k \times 10^{6}$ \\
\hline \multirow[t]{3}{*}{5.00} & 0.1182 & 0.7602 & 0.7552 & 0.1189 & 0.642 & \multirow[t]{3}{*}{25.50} & 14.39 \\
\hline & 0.1179 & 0.7510 & 0.7545 & 0.1179 & 0.635 & & 14.21 \\
\hline & 0.1657 & 0.8023 & 0.7997 & 0.1704 & 0.633 & & 14.20 \\
\hline \multirow[t]{5}{*}{4.00} & 0.1714 & 0.7714 & 0.7700 & 0.1634 & 0.603 & \multirow[t]{5}{*}{24.02} & 14.29 \\
\hline & 0.1037 & 0.7083 & 0.7116 & 0.1078 & 0.604 & & 14.30 \\
\hline & 0.1966 & 0.8002 & 0.8018 & 0.1940 & 0.607 & & 14.36 \\
\hline & 0.2209 & 0.8235 & 0.8239 & 0.2216 & 0.603 & & 14.28 \\
\hline & 0.2096 & 0.8089 & 0.8115 & 0.2069 & 0.602 & & 14.25 \\
\hline \multirow[t]{3}{*}{3.00} & 0.1594 & 0.6941 & 0.6913 & 0.1594 & 0.533 & \multirow[t]{3}{*}{20.92} & 14.42 \\
\hline & 0.1554 & 0.6893 & 0.6862 & 0.1585 & 0.531 & & 14.40 \\
\hline & 0.2222 & 0.7558 & 0.7524 & 0.2222 & 0.532 & & 14.41 \\
\hline
\end{tabular}

Mean $k=-(14.32 \pm 0.11) \times 10^{6}$.

Maximum deviation from mean, 0.77 per cent.

The Susceptibility of Bismuth.-As a first test of the method a number of observations were taken on the susceptibility of bismuth. The bismuth used was merely the purest obtainable from Kahlbaum, but great care was taken to avoid contaminating it with paramagnetic material, especially iron, while preparing it for use, it 
being cast in glass tubes and turned with a carborundum crystal as tool. The susceptibilities determined for a number of specimens, prepared by casting in a glass tube and allowing to cool freely in air, are as follows:

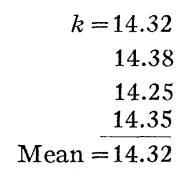

We also attempted to determine whether the susceptibility of bismuth could be altered by the heat treatment used in preparation-i.e., sudden chilling after casting, cooling in air (rather rapid) or long slow annealing. Some difference might perhaps be expected due to the first two and the last methods of preparation. To this we cannot as yet give a definite answer, though there is indication that such an effect exists, for the mean of the values of $k$ for specimens cooled in air, and of those slowly cooled in the furnace from the molten state are:

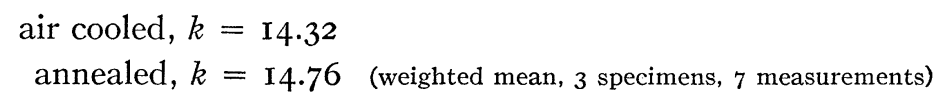

That this difference is not due to the presence or absence of blowholes in one case or the other is shown by the fact that the average density of the air-cooled specimens is 9.82 , of the slow-cooled, 9.83 . This matter of the effect of heat treatment (crystal structure) will be followed further. Our average value of $k$ for air-cooled bismuth agrees well with the mean of previous observations, and the individual measurements are somewhat more consistent.

The Susceptibility of Bismuth-Tellurium and Bismuth-Thallium Alloys.-Aside from the work of Laws and Clifford, relatively little work has been done on the relation between diamagnetic susceptibility and composition of alloys. We selected the above group of alloys for work for the reasons, first, that their chemical and (other) physical properties were more or less known, and, second, because they both indicate, by their melting points and other physical properties, the existence of definite compounds between the constituents.

The tellurium used was obtained from Professor Lenher, of the 
Chemistry Department of the University of Wisconsin, and was prepared with great care under his direction by Dr. Charles Tibbals. The thallium was Schuckhardt's chemically pure; the bismuth, Kahlbaum's chemically pure (the same as that used in the measurements on the susceptibility of bismuth above). Since tellurium and thallium oxidize very rapidly when heated in air the alloys were fused in a reducing atmosphere of hydrogen in an electric furnace, the temperature in which was determined by a thermopile. Great care was exercised in the preparation of the alloys to introduce no impurities. The tellurium as obtained was in a powdered form which melts at a higher temperature than the solid material. In order, then, to reduce the losses by vaporization in the fusing process this powder was first melted together. After cooling, the solid mass could be conveniently broken into pieces, for the preparation of the alloys, by pounding in a mortar. The thallium was cut into chips with a knife and boiled in dilute $\mathrm{HCl}$ to remove abraded iron. Both tellurium and thallium alloy very well with bismuth and the compositions calculated from the weights of the materials originally put into the alloy are known to check very closely with chemical analyses (Monkemeyer and Chikashige). ${ }^{1}$ The alloys were, therefore, not analyzed after the measurement of their susceptibilities. The alloys were turned up in a lathe to the size required by a tool formed from a carborundum crystal. This eliminates the effect, noted by Clifford, ${ }^{2}$ of iron abraded from an ordinary tool.

The susceptibilities for the alloys with tellurium are given in Table II.; for thallium in Table III. The values given are the mean of two or more observations except in the case of nos. 21 to 25 , inclusive, for which but one observation on each was made The measurements were made at temperatures varying from $20^{\circ}$ to $25^{\circ} \mathrm{C}$. Tables II. and III. are plotted in the curves of Figs. 4 and 5 .

The susceptibility of bismuth is taken from our results given above $\left(-14.32 \times 1^{-6}\right)$. The susceptibility found for thallium (mean of two specimens and three observations $-2.46 \pm 0.06 \times$ $\left.10^{-6}\right)$ is considerably lower than that found by Königsberger ${ }^{3}$

${ }^{1}$ Zeitschrift f. anorg. Chem., 46, 4I5, I905, and 5r, 328, I906.

${ }^{2}$ Loc. cit.

${ }^{3}$ Königsberger, Wied. Ann., 66, 698, x 892. 
TABLE II.

Bismuth Tellurium Alloys.

\begin{tabular}{c|c|l|c}
\hline $\begin{array}{c}\text { Specimen } \\
\text { Number. }\end{array}$ & $\begin{array}{c}\text { Per Cent. Bi. } \\
\text { Weight. }\end{array}$ & \multicolumn{1}{|c|}{ Method of Preparation. } & Ave. $k \times$ 10 $^{8}$ \\
\cline { 2 - 4 } 14 & 00.00 & Cast in glass tube. & 2.26 \\
33 & 10.00 & From No. 16 by adding Te. & 1.87 \\
16 & 25.00 & From No. 15 by adding Te. & 2.14 \\
15 & 52.14 & Melting together. & $2.89^{2}$ \\
35 & 52.14 & From No. 33 by adding Bi. & 2.60 \\
17 & 75.00 & From No. 15 by adding Bi. & 4.48 \\
21 & 95.00 & From No. 17 by adding Bi. & 8.25 \\
Ac & 100.00 & Fusing from Kahlbaum. & 14.32 \\
\hline
\end{tabular}

TABLE III.

\begin{tabular}{c|c|c|c}
\multicolumn{3}{c}{ Bismuth Thallium Alloys. } \\
\hline $\begin{array}{c}\text { Specimen } \\
\text { Number. }\end{array}$ & $\begin{array}{c}\text { Per Cent. Bi. } \\
\text { Weight. }\end{array}$ & \multicolumn{1}{|c}{ Method of Preparation. } & Ave. $-k \times$ ro $^{\mathbf{6}}$ \\
\cline { 2 - 3 } 18 & 0000 & Cut directly from stick. & 2.47 \\
25 & 0000 & Cut directly from stick. & 2.45 \\
36 & 10.90 & From No. 23 by Adding Tl. & 1.49 \\
23 & 25.00 & Melting together. & 0.87 \\
20 & 47.53 & Melting together. & 1.38 \\
37 & 56.00 & From No. 20 by adding Bi. & 1.57 \\
19 & 63.00 & Melting together. & 1.30 \\
26 & 63.00 & Melting together. & 0.97 \\
38 & 67.00 & From No. 37 by adding Bi. & 2.42 \\
22 & 80.34 & From No. 19 by adding Bi. & 6.08 \\
24 & 95.00 & Melting together. & 11.40 \\
$A_{c}$ & 100.00 & Melting from Kahlbaum. & 14.32 \\
\hline
\end{tabular}

$\left(-4.6 \times \mathrm{IO}^{-6}\right)$, the only other experimenter who has made measurements on thallium, so far as we are aware. This may indicate that out thallium was less pure than Königsberger's though he makes no statement concerning the source or purity of his material.

The susceptibility found for tellurium (one specimen and mean of two observations $-2.26 \pm 0.05 \times \mathrm{IO}^{-6}$ ) is slightly higher than the result of Königsberger, ${ }^{3}\left(-2 . \mathrm{I} \times \mathrm{IO}^{-6}\right)$. Other experimenters have found for tellurium $-.006,{ }^{4}-\mathrm{I} .6^{5}$ and $\left.-\mathrm{I} .9 \times 10^{-6}\right)$. Our

1 The composition for numbers $\mathrm{I} 5$ and 35 was $\mathrm{Bi}_{4} \mathrm{Te}_{3}$.

${ }_{2}^{2}$ The composition for numbers $I 9$ and 26 was $\mathrm{Bi}_{5} \mathrm{Tl}_{3}$.

${ }^{3}$ Königsberger, Wied. Ann., 66, 698, 1892.

${ }^{4}$ St. Meyer, Wied. Ann. 68, 375; 69, 236, I899.

${ }^{5}$ Von Ettinghausen, Wied. Ann., I7, 272.

${ }^{6}$ Curie, Comptes Rendus, II5 and Ix6, I892. 
tellurium was very pure so that the value obtained is probably very good.

That the two determinations on the compound $\mathrm{Bi}_{5} \mathrm{Tl}_{3}$ (Fig. 4) do not check very closely probably indicates that the compound is on a steep maximum of the curve. This deviation may also be partially accounted for by the fact (as shown by Table II.) that

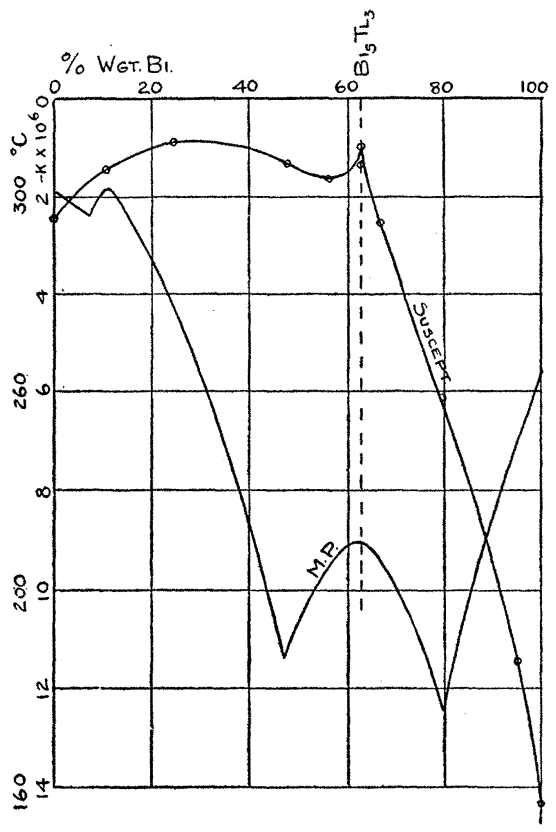

Fig. 4.

Bismuth thallium alloys.

one of the specimens was prepared after four successive fusions from other alloys, and here its assumed composition may have been somewhat in error. The points for the compound $\mathrm{Bi}_{2} \mathrm{Te}_{3}$ (Fig. 5) lie on a smooth curve through the other points. It is possible that the points should be on a small cusp similar to that obtained for $\mathrm{Bi}_{5} \mathrm{Tl}_{3}$. Its detection is, however, either beyond the limits of accuracy of the method or would require the determination of a great number of points in the neighborhood of the cusp.

The melting-point curves in Figs. 4 and 5 are taken from the 
work of Monkemeyer and Chikashige; ${ }^{1}$ those of thermo-electric force and electrical conductivity for tellurium from Haken. ${ }^{2}$ No corresponding data on thallium are available at present.

It is interesting to note the difference in the behavior of the tellurium and thallium alloys at the point of chemical combination. The thallium shows a well-defined cusp, while the tellurium curve is quite smooth, in spite of the fact that other physical properties show marked discontinuities. That thallium is a metal and tel-

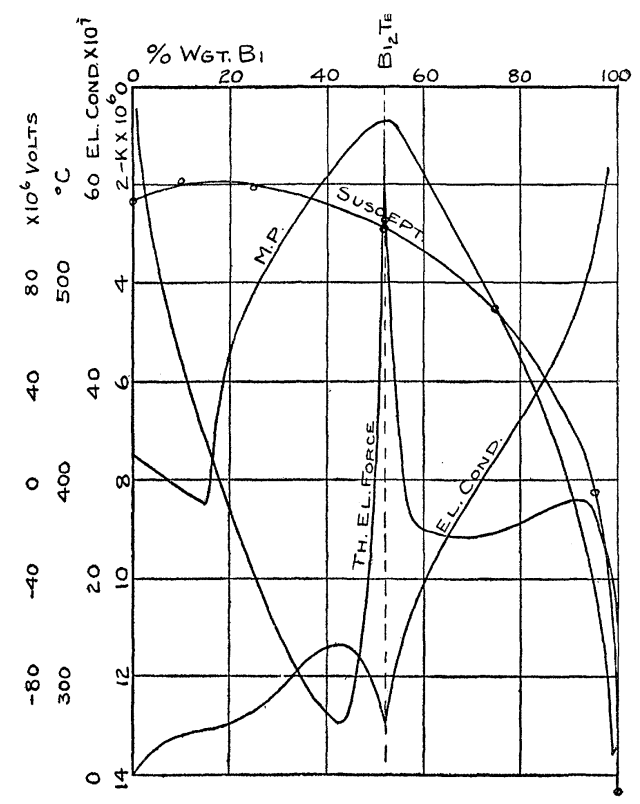

Fig. 5.

Bismuth tellurium alloys.

lurium on the border line between metals and non-metals may or may not be a factor in the explanation. Chemists generally accept maxima on the melting-point curves as good evidence of chemical combination. On this basis we have two chemical compounds in the bismuth-thallium alloys (Fig. 4). Strangely enough we have unique magnetic properties for $\mathrm{Bi}_{5} \mathrm{Tl}_{3}$ but not for the other compound, and not for $\mathrm{Bi}_{2} \mathrm{Te}_{3}$. Other groups of alloys will be studied with this point in mind.

${ }^{1}$ Loc. cit.

${ }^{2}$ Haken, Ber. d. Deutsch. Phys. Gesell., March I5, rgro. 


\section{SUMMARY.}

I. A null method for measuring magnetic susceptibility is described and its accuracy discussed.

2. The mean value $k=\mathrm{I} 4.32$ is obtained for pure bismuth.

3. Preliminary results on the effect of heat-treatment (crystal structure) on the susceptibility of bismuth are given.

4. The curves connecting $k$ and percentage composition for the series of Bi-Te alloys is given, showing no peculiar magnetic properties for the compound $\mathrm{Bi}_{2} \mathrm{Te}_{3}$.

5. The corresponding curve for Bi-Th alloys is also determined and does show peculiar magnetic properties for the compound $\mathrm{Bi}_{5} \mathrm{Tl}_{3}$.

UNIVERSITY OF WisCONSIN,

Department of Physics,

November, I9ro. 